of the reticuloendothelial system. It is inherited as an autosomal recessive and to date 10 patients have been described. In all patients the tonsils or tonsillar remnants show a pathognomonic yellow-orange colouration and in four patients, including the one we have studied, peripheral neuropathy has been present. Death from presumed myocardial infarction has been reported in a 48-year-old man. Serum cholesterol and phospholipid levels are decreased but triglyceride is increased and the serum is turbid even in the fasting state. In our patient we have demonstrated that the proportion of triglyceride is increased in all the lipoprotein fractions including the small amount of alphalipoprotein. Levy et al. [1] have shown that in this condition serum triglyceride can be influenced by dietary carbohydrate and in our patient long-term carbohydrate restriction resulted in some reduction in serum triglyceride levels (from $400 \mathrm{mg} / 100 \mathrm{ml}$ to about $200 \mathrm{mg} / 100 \mathrm{ml}$ ). In an attempt to lower triglyceride levels further chlorophenoxyisobutyrate was given; triglyceride levels were not lowered but there has been a significant increase in total cholesterol concentration (from $50 \mathrm{mg} / 100 \mathrm{ml}$ to $111 \mathrm{mg} / 100 \mathrm{ml}$ ) with a proportionate increase in beta-lipoprotein cholesterol (from $10 \mathrm{mg} / 100 \mathrm{ml}$ to $45 \mathrm{mg} / 100 \mathrm{ml})$. It is not known whether this rise is due to mobilisation of cholesterol from tissues; there has been no marked change in the clinical features over a 2 -year period.

1. LeVy, R.I.; LeES, R.S. and Fredrickson, D.S.: J. clin. Invest. 45: 531 (1966).

\title{
INDEX OF ABSTRACTS
}

(Numbers following entries refer to abstract number)

A-beta-lipoproteinemia 68

Acid-base metabolism 18

ACTH 24

Agathopoulos, A. 12

Aicardi, G. 23

Alagille, D. 54

Aldolase, liver 22

Amino acid metabolism 31

Amino acids 55

Amino acids, metabolism 55

$\delta$-Aminolevulinic acid 56

Anderson, C. M. 42

Anemia, congenital hypoplastic 59

Anemia, hemolytic 32, 60

Anemia, hypoplastic 33

Aneuploidy, XYY 17

Argininosuccinate synthetase 10

AVERY, M.E. 3

BÄGHr, T. 45

BAKKEN, A.F. 50

BAptistal, R. 51

Bettens, W. 22

BiCKel, H. 58

BIER, H. 41

Bile acid metabolism 49

Bilirubin 50

Binoux, M. 24

Binz, H. 45

Blood glucose 5

BoDÁ, D. 6

Bonnassieux, M. 14, 51

Boys 17

BRANDT, N. J. 32

Bronchitis 29

Bronchopulmonary dysplasia 8

Burgio, G.R. 26

Burkitt's tumor 16

Buyle, M.L. 48

Calcium 4, 34

Carditis, rheumatic 26

Cartilage 4

Casteels-van Daele, M. 22
Catecholamines 12, 56

Catechol-O-methyltransferase 12

Celander, O. 1

Celiac disease 47

Central nervous system 13

Children 28, 55

Cholestasis 53

Cholesterol 54

Chromosomes 16, 17

Collagen, skin 23

Coma, hepatic 27

Compliance, lung 7

Copper metabolism 58

CoRbeel, L. M. 22

Corticosteroids 3

Cotre, J. 51

Courtecuisse, V. 54

Crance, J.P. 7

Crigler-Najjar syndrome 51

Cser, A. 5

Guisinier-Gleizes, P. 4

Cystathionase 19

DELemos, R.A. 3

Delinquents 17

Developmental biochemistry 13

Diabetes mellitus 67

Diarrhea 36

Diarrhea, congenital, chloride wasting 48

Dipeptidases, intestinal 38

DONHOFFER, H. 5

DonnadieU, M. 24

Dwarfism, primordial 25

Dysmature 5

EGK, E. 6

EGGERMONT, E. 22

Enterokinase 41

Enterokinase deficiency 39, 40

Enzyme 21

Enzyme induction 10

Enzymes 12

Erythroblastosis 60
Erythrocyte 60

Escherichia coli 46

Exchange transfer 27

Extracorporeal support 27

Factor II 11

Factors VII-X 11

Familial protein intolerance 31

Fat transport, intestinal 43

FERNANDES, J. 21

FERRIER, P.E. 17

FERRIER, S.A. 17

Fetal 38

Fetus 1, 3, 10

Fondu, P. 33

FrançOIS, R. 20, 51

Free fatty acid 5,67

FRÉZAL, J. 68

Fructose 22

Galactose 37

Ganz, R. 8

Gastroenteritis, Escherichia coli 46

Gatti, R. 23

Gaull, G.E. 19

GenTz, J. 56

GHADIMI, H. 15

GIRARD, F. 24

Gitzelmann, R. 45

GJEssing, L. 55

GLADTKE, E. 62

Glucagon 20

Glucose 37

Glucose metabolism 5

Glucose 6-phosphatase 20, 21

Glucosuria, renal 37

Glucuronyltransferase 51

Gluten 47

Glycogen 14

Glycogenosis 20, 21

HADORN, B. 39, 40

HaLVORSEN, S. 55

Hanel, H. 32 
Heart-reactive serum factors 26

Heart, sounds 1

Heim, T. 5

Hemoglobin 61

Hepatic glycogen storage

disease 21

Hepatitis 27

Herschkowitz, N. 13

Hiltunen, P. 30

Holzes, A. 46

Homocystinuria 19

Homolanthionine 19

HÜRTER, P. 60

Hyperammonemia 31

Hyperbilirubinemia 51

Hyperbilirubinemia, neonatal 52

Hypercalcuria, idiopathic 4

Hypercholesterolemia 54

Hypercholesterolemia, familial 65

Hyperlipidemia 30

Hypertriglyceridemia 66

Hyperuricemia 15

Hypopituitarism 24

Hypoxia 1

Immunology 26

Infancy 49

Infant 14, 27

Infants 7, 28, 55, 63

Insensible water loss 2

Insulin 20

Intestine 62

Intrahepatic cholestasis 53

Iron metabolism 62

JEAN, R. 27

KarLSSON, B. 38

KEKOMÄKI, M. 31

Ketosis 21

KNELSON, J.H. 3

KOTAS, R.V. 3

Kouvalainen, K. 30

KowalewsKI, S. 53

KuHNAST, M. 7

Kwashiorkor 44

Lactate 5

LAMPERT, F. 16

LeBove, F. 4

Leigh's encephalopathy 14

Lesch-Nyhan disease 15

Leukocyte 9

Leukocytes 17

LINDBERG, T. 38

LiNDBLAD, B. 56

LindenManN, J. 45

LINDQUIST, B. 36

LINDSTEDT, S. 56

Lipid metabolism 67

Lipoproteins, serum 64

Liver $10,12,14,49,52,54,58$

Liver diseases 55

Liver, lysosomal diseases 57

LLOYD, J. K. 64, 69

LOEB, H. 33, 48

Lung 2, 3

Lymphocytes 16
MaAtela, J. 30

Magnesium 34

Malabsorption, glucose-galactose 37

Malabsorption, intestinal 42

Malabsorption, protein 35

Malabsorption, sugar 36

Malnutrition 44

MAsera, G. 11

Mathieu, H. 4

Mathieu, M. 14, 51

MatsanioTts, N. 12

Medovy, H. 28

Meeuwisse, G. 37

MEstýn, J. 5

$\beta$-Methylcystathionine 19

Milner, R. D. G. 44

Moe, P.J. 63

Monosodium glutamate 15

Moreau, P. 20, 51

Mucopolysaccharidosis 23

Mucosa, small intestine 42

Murányi, L. 6

Myelin 13

Neonatal 38

Newborn 5, 12, 62

Newborn, rabbits 11

Newborns 2, 6, 7, 9, 60

Nicolopoulos, D. 12

Nielson, J. 17

NIKKARI, T. 30

NoRdio, S. 23

Norman, A. 49

ÖCKerman, P.A. 57

Olafsson, A. 8, 52

Orsini, A. 61

Osteopetrosis 63

Osteoporosis 4

Oxygen 5

Pancreas, pseudocyst 28

Pantlitschko, M. 18

Parathyroid extract 34

Parvinen, T. 30

PAUNIER, L. 34

Perheentupa, J. 31

Peritoneal dialysis 6

Perletti, L. 11

Perrimond, H. 61

Petrt, P. 48

Pfeiffer, R. 25

Pham-HuU-trung, M.T. 24

Phenobarbital 51

Phenobarbitone 52

Phospholipids 60

Prepsz, A. 48

Pierson, M. 7

PikaAR, N.A. 21

Polonovskr, C. 41

Premature 3, 5, 10, 12

Prematures 8

Purine synthesis 15

Pyridoxine 19

Pyruvate kinase 32

RÄIHÄ, N.C. R. 10

REQUIEN 20
Respiratory distress syndrome $6,7,8$

Rey, J. 43, 66

REY, J.P. 34

Rheumatic fever 26

RiEU, D. 27

Rottauwe, H.W. 53

RuitTon 20

RÜTER, E. 58

SAlte, B. 14

SCHAFFNER, F. 19

SGHÄRER, H. 58

SaHIMPFF, R. M. 24

SCHRÖTER, W. 60

Sahwartz, A. L. 10

Semenza, G. 45

SERENI, F. 11

Sereni, L. 11

SEveri, F. 26

Shermeta, D.W. 3

SHINER, M. 47

SHMERLING, D. H. 47

Signer, E. 52

Simkó, I. 29

Sjölin, S. 59

SkJaeveland, A. 63

Sodium 4

Sterky, G. 67

STRANDVIK, B. 49

STURMAN, J.A. 19

Sucrase-isomaltase, intestinal 45

Surfactant 3

Tangier disease 69

TARLOW, M.J. 40

Tay-Sachs disease 23

Trmmermans, J. 22

Tommasi, M. 14

Trypsinogen deficiency, pseudo 41

Tyrosinemia 56

Tyrosine transaminase 10

UGLIENGO 20

VacGaro, R. 26

VAINSEL, M. 48

VAN DE KAMER, J.H. 35

VAN DE VELDE, G. 33

Vanillic mandelic acid 12

VERT, P. 7

Vest, M. 52

VISAKORPI, J. K. 31

Vovan, L. 61

Werjers, H.A. 35

WEIPPL, G. 18

Weisser, K. 8, 52

Wilson's disease 58

WrTMer, G. 4

WoLFF, O.H. 65

WRANNE, L. 59

WYLER, F. 8

WYss, M. 34

Xanthou, M. 9

ZETTERSTRÖM, R. 49, 56

ZWEYMÜLLER, E. 2 